medRxiv preprint doi: https://doi.org/10.1101/2021.07.28.21261239; this version posted July 30, 2021. The copyright holder for this preprint

\title{
Molecular typing of breast cancer in Northern Henan Province
}

\author{
Hui Zhao ${ }^{1}$, Haibin $\mathrm{Ma}^{2}$, Bangze Chen ${ }^{2}$, Yahui $\mathrm{Li}^{3}$, Junzheng Yang ${ }^{2 *}$ \\ ${ }^{1}$ Xinxiang Central Hospital, the Fourth Clinic al College of Xinxiang Medical University, \\ Xinxiang, 453000, China \\ ${ }^{2}$ Bioland Laboratory (Guangzhou Regenerative Medicine and Health Guangdong Laboratory), \\ Guangzhou, 510700, China \\ ${ }^{3}$ Guanzhou high-tech pharmaceutical and food technical school, \\ Guangzhou, 511400, China \\ *Correspondence to Junzheng Yang (yangjunzheng606403@163.com), Bioland \\ Laboratory(Guangzhou Regenerative Medicine and Health Guangdong Laboratory), \\ Guangzhou, 510700, China
}

\begin{abstract}
Objective: Investigating and analyzing the clinical features of breast cancer patients in Northern Henan Province, measuring expression of biomarkers (ER, PR, HER2 and $\mathrm{Ki}-67)$ and classified the molecular typing of breast cancer patients, to understand the molecular typing distribution and correlation between biomarkers of breast cancer patients in North Henan Province, which may provide the information for the local oncologist to make sound treatment plans.
\end{abstract}

Methods: We collected the clinical data of breast cancer patients in Xinxiang Central Hospital from 2016 to 2021, those data was classified by gender and pathological types of breast cancer patients; and we also measured and analyzed the expression of breast cancer related biomarkers (ER, PR, HER2 and Ki-67) by immunohistochemistry, and based on expression of these biomarkers, the molecular typing of breast cancer were also classified.

Results: 3210 cases breast cancer patients were collected in this study; there were 3205 female patients and 5 male patients, accounting for $99.84 \%$ and $0.16 \%$ in total breast cancer patients, respectively. Classification according to pathological conditions of breast cancer patients, there were 2761 cases patients with invasive ductal carcinoma, accounting for $86.01 \%$ in total breast cancer patients, and then mucinous NOTE: This preprint reports new research that has not been certified by peer review and should not be used to guide clinical practice. adenocarcinoma (109/3210, 3.40\%), lobular carcinoma (106/3210, 3.30\%), ductal 
medRxiv preprint doi: https://doi.org/10.1101/2021.07.28.21261239; this version posted July 30, 2021. The copyright holder for this preprint

carcinoma in situ $(75 / 3210,2.34 \%)$, papillary carcinoma $(61 / 3210,1.90 \%)$, intraductal carcinoma (40/3210,1.25\%), myeloid carcinoma (27/3210, 0.84\%); There were also including some rare breast cancer types including cribriform carcinoma $(6 / 3210$, $0.19 \%)$, lymph node metastasis $(7 / 3210,0.22 \%)$, occult breast carcinoma $(5 / 3210$, $0.14 \%)$, invasive carcinoma $(5 / 3210,0.14 \%)$, squamous cell carcinoma (3/3210, $0.09 \%)$, fibroadenoma (3/3210, 0.09\%), pleomorphic carcinoma $(2 / 3210,0.06 \%)$. Classification according to molecular typing of breast cancer, the number of breast cancer patients with Luminal A type $[\mathrm{ER}(+) / \mathrm{PR}(+)$ HER2(-)Ki67<14\%] were 207 cases, accounting for $6.45 \%$ in total breast cancer patients, the number of breast cancer patients with Luminal B type I [ER(+)/PR(+) HER2(-)] were 243 cases, accounting for $7.57 \%$ in total breast cancer patients, the number of breast cancer patients with Luminal B type II $[(\operatorname{ER}(+) / \mathrm{PR}(+) H E R 2(+)$ any Ki67] was 254 cases, accounting for $7.91 \%$ in total breast cancer patients, and the number of Triple-negative breast cancer (TNBC) were 390 cases, accounting for $12.15 \%$ in total breast cancer patients.

The average expression rate of $\mathrm{Ki}-67$ in ER (+) and/or PR (+) breast cancer patients was $20.39+27.33 \%$, while the average expression rate of $\mathrm{Ki}-67$ in $\mathrm{ER}(-) / \mathrm{PR}(-)$ breast cancer patients was $36.35 \%+30.14 \%$, and the difference between two patients was significant $(\mathrm{p}=0.0021)$; the average expression rate of Ki-67 in HER2 positive breast cancer patients was $23.01 \%+21.96 \%$, the average expression rate of $\mathrm{Ki}-67$ in HER2 negative breast cancer patients was $29.44 \%+24.16 \%$, and there was no significant difference between the two groups $(\mathrm{P}=0.2589)$.

The main treatment methods of breast cancer patients in Northern Henan Province were antitumor drugs and chemotherapy, the results showed that $87.29 \%$ patients were treated by chemotherapy; and high frequency anti-tumor drugs used for breast cancer treatment were Epirubicin (1527/3210, 47.57\%), Cyclophosphamide (1172/3210, $36.51 \%)$, Paclitaxel (1141/3210, 35.55\%), Tamoxifen (912/3210, 28.41\%).

Conclusions: The main pathological type of breast cancer are invasive ductal carcinoma, and the main treatment methods of breast cancer patients in Northern Henan Province were antitumor drugs and chemotherapy. In the four kinds of molecular typing of breast cancer, the incidence rate of TNBC is highest compared 
medRxiv preprint doi: https://doi.org/10.1101/2021.07.28.21261239; this version posted July 30, 2021. The copyright holder for this preprint

with Luminal B type $\operatorname{II}[(\operatorname{ER}(+) / P R(+) H E R 2(+)$ any Ki67], Luminal B type I $[\operatorname{ER}(+) / P R(+) H E R 2(-)]$ and Luminal A type $[\operatorname{ER}(+) / P R(+)$ HER2(-) Ki67<14\%]; these results may provide some suggestions for the local oncologist.

Keywords: breast cancer; molecular typing; TNBC; Northern Henan Province.

\section{Introduction}

Breast cancer is one of the main causes for threatening women's health worldwide. There is a high mortality rate no matter in developed countries or developing countries ${ }^{[1]}$. Compared with other countries, the incidence of breast cancer in China is relatively low; but with the environmental change and economic development, the number of breast cancer patients in China's city and rural areas has been increasing in the past several years, diagnosis and treatment of breast cancer is becoming an is sue that needs to attention.

Breast cancer is a highly heterogeneous cancer disease at the molecular level. With the development of science and technology and the in-depth study of breast cancer, scientists have found that some biomarkers such as estrogen receptor (ER), progesterone receptor (PR), human epidermal growth factor receptor-2 (HER2), and Ki-67 (proliferation marker) are not only highly associated with tumor growth, invasion, metastasis, recurrence, and they also has important guiding significance in the prediction and treatment of breast cancer ${ }^{[2]}$. Some articles have demonstrated that ER positive patients are generally treated with hormone therapy, and has good therapeutic effect; overexpression of HER2 in breast cancer patients is often closely related to invasive tumor types and poor clinical outcomes ${ }^{[3]}$; Ki-67, as a nuclear protein associated with cell proliferation, can be used as a prognostic marker for multiple malignancies including breast cancer $^{[4]}$. Therefore, molecular typing of breast cancer is not only an important reference for personalized treatment of breast cancer, but also a good development direction for breast cancer treatment.

Breast cancer is usually divided into four subtypes according to breast cancer molecular typing method, including Luminal A type $[\mathrm{ER}(+) / \mathrm{PR}(+) / \operatorname{HER} 2(-)$ Ki67<14\%], Luminal B type I (ER(+)/PR(+)HER2(-)/Ki67>14\%], Luminal B type II $[\operatorname{ER}(+) / \mathrm{PR}(+) / \mathrm{HER} 2(+)$ any Ki67 $]$, and TNBC[(ER(-)/PR(-)/HER2(-)]. Luminal A type 
medRxiv preprint doi: https://doi.org/10.1101/2021.07.28.21261239; this version posted July 30, 2021. The copyright holder for this preprint

breast cancer, also known as hormone dependent breast cancer, is sensitive to endocrine therapy with good prognosis, but poor sensitivity to chemotherapy; is not sensitive to endocrine therapy, but endocrine therapy has a better survival rate for Luminal B type breast cancer. TNBC is the most studied subtype in breast cancer, it is not sensitive to endocrine therapy and HER2 treatment, and the five year survival rate is the lowest in the four kinds of subtypes of breast cancer.

In this study, we collected 3210 cases of breast cancer in Northern Henan Province, classified the data by gender and pathological types of breast cancer patients, and we also measured and analyzed the expression of breast cancer related biomarkers (ER, PR, HER2 and Ki-67) by immunohistochemistry, based on expression of these biomarkers, the molecular subtypes of breast cancer were also classified, which will lay the foundation for prevention and treatment of breast cancer in North Henan Province.

\section{Research subjects}

The clinical information of 3210 breast cancer patients were collected from 2016 to 2021 at Xinxiang Central Hospital in Northern Henan Province. The medical information including gender, expression of ER and PR, Ki-67 and HER2 were measured and recorded. The study was approved by Ethics Committee of Xinxiang Central Hospital. The informed consent was obtained from all patients. The procedures performed in this study involving human participants were in accordance with the Helsinki Declaration of 1975, as revised in 2008.

\section{Research Methods}

In this article, we collected the clinical data of 3210 breast cancer patients in Xinxiang Central Hospital from 2016 to 2021, the data was classified by gender and pathological types of breast cancer patients; and we also measured and analyzed the expression of breast cancer related biomarkers (ER, PR, HER2 and Ki-67) by immunohistochemistry, and based on expression of these biomarkers, the molecular subtypes of breast cancer were also classified.

Expression of ER, PR, HER-2 and Ki-67 was detected by immunohis tochemistry staining, tissue species were formalin fixed, dehydrated in alcohol and embedded in 
medRxiv preprint doi: https://doi.org/10.1101/2021.07.28.21261239; this version posted July 30, 2021. The copyright holder for this preprint

paraffin. Immunostaining was performed according to the streptavidin-biotin peroxidase complex method, using monoclonal antibodies against ER, PR, HER-2 and Ki-67. Briefly, tissue species were cut into a thickness of $4-5 \mu \mathrm{m}$, and dried 1 hour at $60^{\circ} \mathrm{C}$ in a fresh slide. And then All the slides were deparaffinized in xylene, rehydrated through alcohol, and washed in phosphate-buffered saline. This buffer was used for all subsequent washes. slide for ER, PR, HER-2 and Ki-67 detection were heated in a microwave oven twice for $5 \mathrm{~min}$ in citrate buffer ( $\mathrm{pH}$ 6.0). Monoclonal antibodies (at a dilution of 1:500) ER, PR, HER-2 and Ki-67 were used respectively as the primary antibody and incubated overnight at room temperature followed by a conventional streptavidin peroxidase method. Signals were developed with 3, 30-diaminobenzidine for 5 minutes and counter-stained with hematoxylin. The results were scored for the positive signals in tis sue cells. For ER and PR scoring, $>1 \%$ positive staining cells in the breast cancer specimens were considered ER or PR positive expression. For HER-2 scoring, scores 0 and 1 were considered as negative expression, 2 and 3 were considered as positive expression. Ki-67 proliferation level was determined by the percentage of positive cells among the tested cells. For each slide, the pathological testing results were reviewed by two qualified pathologists for interpretation and diagnosis.

\section{Statistical Analysis}

Data are reported as the mean \pm SD. t-test or Fisher test was used to analyze the difference between different disease subtypes, $\mathrm{P}>0.05$ was considered statistical difference.

\section{Results}

A total of clinical information of 3210 breast cancer patients were collected in Xinxiang central hospital from 2016 to 2021. There were 3205 female breast cancer patients and 5 male breast cancer patients, accounting for $99.84 \%$ and $0.16 \%$ in total breast cancer patients, respectively; Classification according to pathological conditions of breast cancer patients, there were 2761 cases patients with invasive ductal carcinoma, accounting for $86.01 \%$ in total breast cancer patients, and then mucinous adenocarcinoma (109/3210, 3.40\%), lobular carcinoma $(106 / 3210,3.30 \%)$, ductal 
medRxiv preprint doi: https://doi.org/10.1101/2021.07.28.21261239; this version posted July 30, 2021. The copyright holder for this preprint

carcinoma in situ $(75 / 3210,2.34 \%)$, papillary carcinoma $(61 / 3210,1.90 \%)$, intraductal carcinoma (40/3210,1.25\%), myeloid carcinoma (27/3210, 0.84\%); There were also including some rare pathological types of breast cancer including cribriform carcinoma $(6 / 3210,0.19 \%)$, lymph node metastasis $(7 / 3210,0.22 \%)$, occult breast carcinoma $(5 / 3210,0.14 \%)$, invasive carcinoma $(5 / 3210,0.14 \%)$, squamous cell carcinoma (3/3210, 0.09\%), fibroadenoma $(3 / 3210,0.09 \%)$, pleomorphic carcinoma $(2 / 3210$, $0.06 \%$ ) (Table 1$)$.

Table 1. Pathological type distribution of breast cancer in 3210 cases of breast cancer patients in North Henan Province

\begin{tabular}{ccc}
\hline Breast cancer type & Cases & Proportion \\
\hline Invasive ductal carcinoma & 2761 & $86.01 \%$ \\
Mucinous adenocarcinoma & 109 & $3.40 \%$ \\
Lobular carcinoma & 106 & $3.30 \%$ \\
Ductal carcinoma in situ & 75 & $2.34 \%$ \\
Papillary carcinoma & 61 & $1.90 \%$ \\
Intraductal carcinoma & 40 & $1.25 \%$ \\
Medullary Breast Carcinoma & 27 & $0.84 \%$ \\
Cribriform carcinoma & 6 & $0.19 \%$ \\
Metastatic carcinoma of lymph node & 7 & $0.22 \%$ \\
Occult breast cancer & 5 & $0.16 \%$ \\
Invasive carcinoma & 5 & $0.16 \%$ \\
Squamous cell carcinoma & 3 & $0.09 \%$ \\
breast fibroadenoma & 3 & $0.09 \%$ \\
Pleomorphic carcinoma & 2 & $0.06 \%$ \\
Total & 3210 & $100.00 \%$ \\
\hline
\end{tabular}

Subsequently, according to the results of immunohistochemistry, we classified and summarized biomarkers expression of ER, PR, HER2, and Ki-67 in 3210 cases breast cancer patients. The number of ER positive and ER negative breast cancer patients 
medRxiv preprint doi: https://doi.org/10.1101/2021.07.28.21261239; this version posted July 30, 2021. The copyright holder for this preprint

were 2379 cases and 831 cases, accounting for $74.11 \%$ and $25.89 \%$ in the total number of breast cancer patients, respectively; the number of PR positive and PR negative breast cancer patients were 2334 cases and 876 cases, accounting for $72.71 \%$ and $27.39 \%$ in the total number of patients respectively; the number of HER2 positive and breast cancer patients were 2561 cases and 649 cases, accounting for $79.78 \%$ and $20.22 \%$ in the total number of breast cancer patients respectively. The number of Luminal A type $[\mathrm{ER}(+) / \mathrm{PR}(+) H E R 2(-) \mathrm{Ki} 67<14 \%]$ breast cancer patients were 207 cases, the number of Luminal B type I [ER(+)/PR(+)HER2(-)Ki67>14\%] breast cancer patients was 243 cases, and the number of Luminal B type II [ER(+)/ PR(+)HER2(+) any Ki67] patients with breast cancer were 254 cases, the number of TNBC was 390 cases, accounting for $6.45 \%, 7.57 \%, 7.91 \%, 12.15 \%$ in the total number of breast cancer patients, respectively (Table 2 and Table 3).

Table 2. Distribution of ER, PR and HER2 in 3210 breast cancer patients in North Henan Province

\begin{tabular}{|c|c|c|c|}
\hline Marker & Type & Cases & Proportion \\
\hline \multirow{2}{*}{ ER } & Positive & 2379 & $74.11 \%$ \\
\hline & Negative & 831 & $25.89 \%$ \\
\hline \multirow{2}{*}{ PR } & Positive & 2334 & $72.71 \%$ \\
\hline & Negative & 876 & $27.39 \%$ \\
\hline \multirow{2}{*}{ HER2 } & Positive & 2561 & $79.78 \%$ \\
\hline & Negative & 649 & $20.22 \%$ \\
\hline
\end{tabular}

Table 3. Molecular typing distribution in 3210 cases of breast cancer in North Henan Province

\begin{tabular}{|c|c|c|c|c|}
\hline Name & & Molecular typing & Cases & Proportion \\
\hline Luminal A & & $\begin{array}{c}\text { ER(+)/PR(+)HER2(-) } \\
\text { Ki67 }<14 \%\end{array}$ & 207 & $6.45 \%$ \\
\hline Luminal B & Type I & $\begin{array}{c}\mathrm{ER}(+) / \mathrm{PR}(+) \mathrm{HER} 2(-) \\
\mathrm{Ki} 67>14 \%\end{array}$ & 243 & $7.57 \%$ \\
\hline
\end{tabular}


medRxiv preprint doi: https://doi.org/10.1101/2021.07.28.21261239; this version posted July 30, 2021. The copyright holder for this preprint (which was not certified by peer review) is the author/funder, who has granted medRxiv a license to display the preprint in perpetuity. It is made available under a CC-BY-NC-ND 4.0 International license.

Type II

TNBC

$$
\operatorname{ER}(+) / P R(+) H E R 2(+)
$$$$
\text { any Ki67 }
$$

ER(-)/PR(-)HER2(-)
254

390
$7.91 \%$

$12.15 \%$

And then we studied the biomarkers relationship among ER, PR and Ki-67. The results showed that the number of $\mathrm{ER}(+)$ and/or $\mathrm{PR}(+)$ breast cancer patients was 2112 cases, accounting for $65.79 \%$ in the total number of breast cancer patients; the number of $\operatorname{ER}(-) / P R(-)$ breast cancer patients was 633 cases, accounting for $19.76 \%$ in the total number of breast cancer patients; the average expression rate of $\mathrm{Ki}-67$ in $\mathrm{ER}(+)$ and/or $\mathrm{PR}(+)$ breast cancer patients was $20.39+27.33 \%$, while the average expression rate of $\mathrm{Ki}-67$ in $\mathrm{ER}(-) / \mathrm{PR}(-)$ breast cancer patients was $36.35 \%+30.14 \%$, there was the statistically significant between two groups $(\mathrm{p}=0.0021)$. We also analyzed relationship between HER2 and Ki-67. The number of HER2(+) breast cancer patients was 2561 cases, accounting for $79.78 \%$ in the total number of breast cancer patients; the number of HER2(-) breast cancer patients was 649 cases, accounting for $21.22 \%$ in the total number of breast cancer patients. The average expression rate of Ki-67 in HER2(+) breast cancer patients was $23.01 \%+21.96 \%$, the average expression rate of $\mathrm{Ki}-67$ in HER2(-) breast cancer patients was $29.44 \%+24.16 \%$, and there was no significant difference between the two groups $(\mathrm{P}=0.2589)$ (Table 4).

Table 4. The correlation among biomarkers ER, PR and Ki-67 in breast cancer patients in Northern Henan Province

\begin{tabular}{cccc}
\hline Biomarkers & Cases & Ki-67 Level $(\%)($ Mean \pm SD $)$ & P-value \\
\hline ER(+)and/or PR(+) & $2112(65.79 \%)$ & $20.39 \pm 27.33 \%$ & 0.0021 \\
ER(-)/PR(-) & $633(19.76 \%)$ & $36.35 \% \pm 30.14 \%$ & \\
HER2(+) & $2561(79.78 \%)$ & $23.01 \% \pm 21.96 \%$ & 0.2589 \\
HER2(-) & $649(21.22 \%)$ & $29.44 \% \pm 24.16 \%$ & \\
\hline
\end{tabular}

Finally, we summarized the treatment methods for breast cancer patients who visited the hospital in the Northern Henan Province, the results showed that $87.29 \%$ of breast cancer patients has been treated by chemotherapy; the highest frequency of 
medRxiv preprint doi: https://doi.org/10.1101/2021.07.28.21261239; this version posted July 30, 2021. The copyright holder for this preprint (which was not certified by peer review) is the author/funder, who has granted medRxiv a license to display the preprint in perpetuity. It is made available under a CC-BY-NC-ND 4.0 International license

antitumor drugs used was Epirubicin (1527/3210, 47.57\%), Cyclophosphamide (1172/3210, 36.51\%), Paclitaxel (1141/3210, 35.55\%), Tamoxifen (912/3210). 28.41\%) (Table 5).

Table 5. Treatment of breast cancer patients in North Henan Province

\begin{tabular}{|c|c|c|}
\hline Types & Cases & $\begin{array}{c}\text { Proportion in } 3210 \\
\text { patients }\end{array}$ \\
\hline Chemotherapy & 2802 & $87.29 \%$ \\
\hline Cyclophosphamide & 1172 & $36.51 \%$ \\
\hline Epirubic in & 1527 & $47.57 \%$ \\
\hline Cisplatin & 153 & $4.77 \%$ \\
\hline Carboplatin & 252 & $7.85 \%$ \\
\hline Paclitaxel & 1141 & $35.55 \%$ \\
\hline Docetaxel & 714 & $22.24 \%$ \\
\hline Vinorelbine & 160 & $4.98 \%$ \\
\hline Gemictabine & 73 & $2.27 \%$ \\
\hline herceptin & 250 & $7.79 \%$ \\
\hline Fluorouracile & 248 & $7.73 \%$ \\
\hline Methotrexate & 62 & $1.93 \%$ \\
\hline Tamoxifen & 912 & $28.41 \%$ \\
\hline xeloda & 228 & $7.10 \%$ \\
\hline Etoposide & 19 & $0.60 \%$ \\
\hline AUSTIN & 7 & $0.22 \%$ \\
\hline Pemetrexed & 9 & $0.28 \%$ \\
\hline Exemestane & 253 & $7.89 \%$ \\
\hline Torremifen & 310 & $9.66 \%$ \\
\hline Letrozole & 271 & $8.44 \%$ \\
\hline Anastrozole & 508 & $15.83 \%$ \\
\hline Norred & 130 & $4.05 \%$ \\
\hline Fulvestrant & 6 & $0.19 \%$ \\
\hline
\end{tabular}


medRxiv preprint doi: https://doi.org/10.1101/2021.07.28.21261239; this version posted July 30, 2021. The copyright holder for this preprint

\begin{tabular}{ccc} 
Methylprogesterone & 24 & $0.75 \%$ \\
Bisphosphate & 97 & $3.02 \%$ \\
Lapatinib & 8 & $0.25 \%$ \\
\hline
\end{tabular}

\section{Discussion}

We collected clinical data of 3210 breast cancer patients in Xinxiang Central Hospital from 2016 to 2021. There were 3205 female patients and 5 male patients, accounting for $99.84 \%$ and $0.16 \%$ respectively; It is found that the existence of male breast cancer in the Northern Henan Province, due to the structural and physiological particularity of male breast, medical staff should pay attention to male psychological factors while treatment. Then we classified breast cancer patients according to the pathological conditions of patients, found that 2761 cases patients were invasive ductal carcinoma, accounting for $86.01 \%$ in the total number of breast cancer patients, 109 cases patients were mucinous adenocarcinoma, 106 cases patients were lobular carcinoma, 75 cases patients were ductal carcinoma in situ; it also existed some rare types of breast cancer: cribriform carcinoma (6/7210, 0.19\%), lymph node metastasis (7/3210, $0.22 \%)$, occult breast cancer $(5 / 3210,0.16 \%)$, invasive carcinoma $(5 / 3210$, 0.16\%), squamous cell carcinoma (3/3210, 0.09\%), fibroadenoma (3/3210, 0.09\%), pleomorphic carcinoma $(2 / 3210,0.06 \%)$. Those data demons trated that invasive ductal carcinoma was mainly pathological type of breast cancer in Northern Henan Province, and multiple types of breast cancer subtypes coexisted, which suggest that clinical staff should judge the disease from multiple perspectives and factors in diagnosis and treatment to improve the treatment effect.

It was reported that the response rate of endocrine therapy in $\operatorname{ER}(+) / \operatorname{HER} 2(+)$ breast cancer patients can be reduced from $84 \%$ to $20 \%$, the response rate of endocrine therapy in ER(-)/HER2(+) breast cancer patients can be reduced from $27 \%$ to 0 ; and expression of HER2 could inhibit the expression of hormone receptor. Therefore, the treatment of Luminal B type breast cancer usually requires endocrine therapy plus chemotherapy ${ }^{[5,6]}$. Because breast cancer with HER2 overexpression is more sensitive to Taxol chemotherapy, chemotherapy combined with biological targeting drugs has a 
medRxiv preprint doi: https://doi.org/10.1101/2021.07.28.21261239; this version posted July 30, 2021. The copyright holder for this preprint

good therapeutic effect on breast cancer with HER2 overexpression ${ }^{[7,8]}$. In this article, we measured and analyzed the expression of ER, PR, HER2 and Ki-67 by immunohistochemistry. We found that the number of ER positive breast cancer patients PR positive breast cancer patients and HER2 positive breast cancer patients, accounting for $74.11 \%, 72.71 \%$ and $79.78 \%$ in the total breast cancer patients respectively. Luminal A type $[\mathrm{ER}(+) / \mathrm{PR}(+) \mathrm{HER} 2(-) \mathrm{Ki} 67<14 \%]$ of breast cancer patients, Luminal B type I [ER(+)/PR(+)HER2(-)Ki67>14\%] breast cancer patients Luminal B type II [ER(+)/PR(+)HER2(+) any Ki67] breast cancer patients, and TNBC were accounting for $6.45 \%, 7.57 \%, 7.91 \%, 12.15 \%$ in the total number of breast cancer patients, respectively. Because the four kinds of molecular typing of breast cancer are different in their tolerance and selectivity to the current treatment methods, it is usually necessary to conduct a relative treatment based on molecular typing, suggesting that the clinical staff should proceed with the diagnosis and treatment according to the molecular typing of breast cancer and the actual situation of the patients.

At the same time, we studied the relationship among ER, PR, HER2 and Ki-67. The average expression rate of $\mathrm{Ki}-67$ in ER positive and/or PR positive breast cancer patients was $20.39+27.33 \%$, while the average expression rate of $\mathrm{Ki}-67$ in patients with $\mathrm{ER}(-) / \mathrm{PR}(-)$ breast cancer was $36.35 \%+30.14 \%$, there was a significant difference between the two groups $(\mathrm{p}=0.0021)$, the data showed that there was a correlation among ER, PR and Ki-67. The average expression rate of $\mathrm{Ki}-67$ in HER2 positive breast cancer patients was $23.01 \%+21.96 \%$, and the average expression rate of $\mathrm{Ki}-67$ in HER2 negative breast cancer patients was $29.44 \%+24.16 \%$, there was no significant difference between the two groups, therefore there was no correlation between HER2 and $\mathrm{Ki}-67(\mathrm{P}=0.2589)$. Those results suggests that clinicians should consider the expression changes of biomarkers such as ER, PR, HER2 and Ki-67 in the diagnosis and treatment of breast cancer.

Finally, we summarized the treatment methods of breast cancer patients in the Northern Henan Province, and found that $87.29 \%$ of breast cancer patients were treated by chemotherapy. The highest frequency of antitumor drugs used for breast cancer treatment was Epirubicin (1527/3210, 47.57\%), Cyclophosphamide (1172/3210, 
medRxiv preprint doi: https://doi.org/10.1101/2021.07.28.21261239; this version posted July 30, 2021. The copyright holder for this preprint

36.51\%), Paclitaxel (1141/3210, 35.55\%), Tamoxifen (912/3210, 28.41\%). indicated that treatment of breast cancer in the northern Henan Province were mainly chemotherapy and antitumor drugs.

\section{Conclusion}

In this article, we summarized the clinical features of 3210 cases breast cancer patients in North Henan Province from 2016 to 2021. It is found that invasive ductal carcinoma is the main pathological type of breast cancer in North Henan Province. In the four kinds of molecular typing of breast cancer, TNBC had a highest incidence rate compared with the other types of breast cancer. it is recommended that reasonable diagnosis and treatment measures should be taken to prevent breast cancer and to improve the therapeutic effect.

\section{Conflict of interests}

The authors declare that they have no competing interests in this article.

\section{Acknowledgements}

None.

\section{Funding}

None.

\section{References}

1. Tao Z, Shi A, Lu C, Song T, Zhang Z, Zhao J. Breast cancer: epidemiology and etiology. Cell Biochem Biophys., 2015, 72(2):333-338.

2. Van Asten K, Slembrouck L, Olbrecht S, et al. Prognostic value of the progesterone receptor by subtype in patients with estrogen receptor-positive, HER2 negative breast cancer. Oncologist, 2019, 24(2):165-171.

3. Hart CD, Migliaccio I, Malorni L, Guarducci C, Biganzoli L, Di Leo A. Challenges in the management of advanced, ER-positive, HER2-negative breast cancer[J]. Nat Rev Clin Oncol., 2015, 12(9): 541-552.

4. Soliman NA, Yussif SM. Ki-67 as a prognostic marker according to breast cancer molecular subtype. Cancer Biol Med., 2016, 13(4):496-504.

5. Matthew J Ellis, Vera J Suman, Jeremy Hoog, Rodrigo Goncalves, et al. Ki67 Proliferation Index as a Tool for Chemotherapy Decisions During and After Neoadjuvant Aromatase Inhibitor 
medRxiv preprint doi: https://doi.org/10.1101/2021.07.28.21261239; this version posted July 30, 2021. The copyright holder for this preprint (which was not certified by peer review) is the author/funder, who has granted medRxiv a license to display the preprint in perpetuity. It is made available under a CC-BY-NC-ND 4.0 International license.

Treatment of Breast Cancer: Results From the American College of Surgeons Oncology Group Z1031 Trial (Alliance). J Clin Oncol., 2017, 35(10):1061-1069.

6. Michael Gnant, Nadia Harbeck, Christoph Thomssen. St. Gallen/Vienna 2017: A Brief Summary of the Consensus Discussion about Escalation and De-Escalation of Primary Breast Cancer Treatment. Breast Care (Basel)., 2017, 12(2):102-107.

7. Michael Gnant, Nadia Harbeck, Christoph Thomssen. St. Gallen 2011: Summary of the Consensus Discussion. Breast Care (Basel)., 2011, 6(2):136-141.

8. Hannah Farmer, Nuala McCabe, Christopher J Lord, Andrew N J Tutt, et al. Targeting the DNA repair defect in BRCA mutant cells as a therapeutic strategy. Nature, 2005, 434(7035):917-921. 